\title{
FERTILIZACIÓN NITROGENADA PARA RENDIMIENTO OBJETIVO DE SORGO GRANÍFERO EN EL SUROESTE DE LA PROVINCIA DE CHACO, ARGENTINA
}

\author{
Cáceres Díaz, Raúl O.; Quintana Gerardo J. R.; Nadal, Nelson y Jimenez, J. O. \\ EEA INTA Las Breñas - rcdiaz@correo.inta.gov.ar
}

\section{RESUMEN}

Es reconocida y recomendada la incorporación del sorgo granífero a la rotación de cultivos por su contribución con el rastrojo al suelo, cobertura que brinda, en la época de barbecho, aporte de materia orgánica. Todo esto contribuye a disminuir la escorrentía. Se realizó un ensayo de fertilización nitrogenada en sorgo granífero, en siembra directa, en la Estación Expe-


Sur y $61^{\circ} 03^{\prime} 30^{\prime \prime}$ Oeste con determinación de nitratos a la siembra para ajuste de dosis de nitrógeno en función de un rendimiento esperado (rendimiento objetivo). Se evaluaron 2 (dos) tratamientos de fertilización, 30 (T1) y 90 (T2) $\mathrm{kg} \mathrm{ha}^{-1}$ de urea, con un contenido de $46 \%$ de N, incorporada al costado de la línea de siembra en el estado V6 y un tratamiento testigo sin fertilizar (T0), para una producción objetivo de 8.000 $\mathrm{kg}$ grano $\mathrm{x}$ ha 1 en $\mathrm{T} 1$ y $10.000 \mathrm{~kg}$ grano $\mathrm{x} \mathrm{ha} \mathrm{h}^{-1} \mathrm{en}$ T2. Se utilizó un diseño en Bloques Completamente Aleatorizados con cuatro repeticiones. El cultivar fue Morgan 109, de ciclo intermedio, sembrado el 22 de enero de 2008, lográndose 100.000 plantas ha ${ }^{-1}$. Durante el ciclo del cultivo se controlaron las plagas condicionantes. La producción de granos del tratamiento T0 fue de 4.942,11 $\mathrm{kg} \mathrm{ha}^{-1}$, del T1 7.345,86 $\mathrm{kg} \mathrm{ha}^{-1} \mathrm{y}$ del T2 $8.518,89 \mathrm{~kg} \mathrm{ha}^{-1}$, no existiendo diferencias significativas entre $\mathrm{T} 1$ y $\mathrm{T} 2(\mathrm{p} \leq 0,05)$. El consumo de agua en el ciclo fue de $301,5 \mathrm{~mm}$, el T0 tuvo una eficiencia en el uso del agua de $16,4 \mathrm{~kg}$ grano por mm agua ${ }^{-1}$ utilizada, el T1 24,4 kg grano por $\mathrm{mm}$ agua $^{-1}$ y el T2 $28,3 \mathrm{~kg}$ grano por $\mathrm{mm}$ de agua ${ }^{-1}$. La eficiencia del uso del fertilizante fue de $80,1 \mathrm{~kg}$ de grano por $\mathrm{kg}$ urea $^{-1}$ utilizada para $\mathrm{T} 1$ y $39,7 \mathrm{~kg}$ de grano por $\mathrm{kg} \mathrm{urea}^{-1}$ utilizada para $\mathrm{T} 2$. El rendimiento de granos, en función de las dosis de urea, respondió a una ecuación polinómica de $2^{\circ}$ Orden $Y=0,673 \mathrm{x}^{2}$ $+100,32 \mathrm{x}+4.942,1$. Los datos obtenidos indican que fue posible incrementar los rendimientos de sorgo granífero a través de la fertilización nitrogenada en V6, utilizando la metodología de rendimiento objetivo bajo las condiciones de experimentación mencionadas a pesar de contar con suelos bien provistos en nitratos a la siembra.
Palabras clave: siembra directa, rotación de cultivos, rastrojo, eficiencia en el uso del agua,

\section{ABSTRACT}

The incorporation of Grain Sorghum to crop rotation as stubble is a well recognized and recommended practice due to the soil coverage offered at the time of fallow. This practice provides organic matter and prevents soil run-off. A test of nitrogen fertilization was done in Grain Sorghum, grown under zero tillage system at the Farming Experimental Station INTA Las Breñas $\left(27^{\circ} 04^{\prime} 70^{\prime \prime}\right.$ South, 61 $61^{\circ} 30^{\prime \prime}$ West). The test determined nitrate levels at sowing time, for gauging nitrogen doses based upon the expected yield (objective yield). Two treatments of fertilization were evaluated, 30 (T1) and 90 (T2) $\mathrm{kg} \mathrm{ha}^{-1}$ of urea, with a $\mathrm{N}$ content of $46 \%$, incorporated alongside the sowing line at the V6 state, and control treatment without fertilizing (T0) for an expected yield of 8,000 $\mathrm{kg}$ grain $\mathrm{x} \mathrm{ha}^{-1}$ in $\mathrm{T} 1$ and $10,000 \mathrm{~kg}$ grain . ha ${ }^{-1}$ in $\mathrm{T} 2$. A completely randomized experimental design with blocks of four repetitions was used. Morgan 109 cultivar with intermediate cycle was seeded on January 22,2008 , which yielded 100,000 plants $x \mathrm{ha}^{-1}$. During the crop cycle, specific pests were controlled. Grain production of the T0 treatment was $4,942.11 \mathrm{~kg} \mathrm{ha}^{-1}$, T1 7,345.86 kg x ha ${ }^{-1}$ and T2 8,518.89 kg.ha- ${ }^{-1}$. There were no significant differences in treatment $\mathrm{T} 1$ and $\mathrm{T} 2(\mathrm{p} \leq 0,05)$. Water consumption in the cycle was $301.5 \mathrm{~mm}$ : T0 had an efficiency of water use of 16.4 $\mathrm{kg}$ grain per mm of water-1 consumption; T1 $24.4 \mathrm{~kg}$ grain by $\mathrm{mm}$ water ${ }^{-1}$ consumption and $\mathrm{T} 2$ had 28.3 $\mathrm{kg}$ grain per $\mathrm{mm}$ of water consumption. The fertilizer efficiency was $80.1 \mathrm{~kg}$ of grain by $\mathrm{kg}$ urea $^{-1}$ applied for $\mathrm{T} 1$ and $39.7 \mathrm{~kg}$ of grain by $\mathrm{kg}$ urea $^{-1}$ applied for T2 respectively. Grain yield, based on the doses of urea, responded to a polinomic equation of second order: $Y=0.673 x^{2}+100.32 x+4,942.1$. The collected data indicates that it is possible to increase yields of Grain Sorghum through nitrogen fertilization in V6, using Objective Yield Methodology, under the mentioned conditions of experimentation. The experiment was carried out in soil with a good provision of $\mathrm{N}$ at seed time. 
Key words: zero tillage, crop rotation, stubble, water use efficiency.

\section{INTRODUCCIÓN}

El sorgo granífero es un cultivo tropical que encuentra fundamentalmente en el Suroeste de la provincia de Chaco las condiciones óptimas para su crecimiento. En dicha región las precipitaciones medias van desde los $1.200 \mathrm{~mm}$. al Este, a los $750 \mathrm{~mm}$ al extremo Oeste con temperaturas de suelo y radiación elevada en los 238 días libres de heladas, promedio de los últimos 4 años, de acuerdo a los datos suministrados por la Estación Agrometeorológica de INTA Las Breñas.

Para la siembra, el sorgo requiere una temperatura de $18^{\circ} \mathrm{C}$ o más, en los primeros $10 \mathrm{~cm}$ del suelo, con emergencia a partir del $3^{\circ}$ ó $4^{\circ}$ día desde la siembra (Melín y Zamora, 2007). Para la zona de Las Breñas, esto ocurre a partir de la primer semana de setiembre (Boletín Agrometeorológico anual, 2008 INTA Las Breñas). En este sentido, es posible su cultivo en una amplitud de días (ventana de siembra), comenzando en forma temprana en setiembre, si hubiera buena humedad en el perfil, cuando la temperatura del suelo a $10 \mathrm{~cm}$ es superior a $20,7^{\circ} \mathrm{C}$ y culminando a fines de enero con una siembra tardía por el riesgo de heladas tempranas.

La provincia de Chaco, de acuerdo a las estadísticas oficiales sembró 33.500 has., con una producción de 67.750 tn en la campaña 05/06 (Dirección de Estadística de la provincia del Chaco, 2006), previendo mantener esta superficie en la presente campaña.

La superficie sembrada a nivel nacional, varió desde 900.000 ha en la campaña $97 / 98$ a 550.000 ha en la campaña $05 / 06$, en la campaña $06 / 07$, la superficie alcanzó las 700.000 ha (SAGPYA, 2009) producto de la demanda como forraje y biocombustibles. En la campaña $07 / 08$ se sembraron 807.000 ha lo que implica $15 \%$ más que el ciclo anterior con una producción de 2.937 .000 tn de grano, con $3.639 \mathrm{~kg} \mathrm{ha}^{-1}$ de rendimiento promedio. En la campaña 08/09 se ha sembrado 825.000 ha.

El aporte de rastrojo es muy importante, considerando un esquema de rotaciones que tienda a proteger el suelo, con aportes medidos de 12 a 17 tn de materia seca $\mathrm{x}$ ha. $\mathrm{x}$ año ${ }^{-1}$, de acuerdo a los resultados de Ensayos de Larga Duración en la Estación Experimental Agropecuaria INTA Las Breñas, Chaco, lo que en siembra directa significa un beneficio considerable para la sostenibilidad del sistema.

Desde el período considerado de rápido crecimiento a partir de los 35 días después de emergencia, las aplicaciones de nitrógeno al costado de la línea de siembra podrían elevar la producción de grano y mejorarían la utilización del nitrógeno, mejorando la eficiencia del cultivo (Vanderlip, 1993). Las pérdi- das gaseosas totales (denitrificación y volatilización) varían según la dosis de nitrógeno aplicada, representando el 7,6\% y $13,8 \%$ para ambos momentos de fertilización (siembra y V6) seis hojas verdaderas desplegadas.

Sin embargo, las emisiones por denitrificación son más importantes en las fertilizaciones a la siembra, explicando el $80 \%$ de las pérdidas gaseosas totales, mientras que la volatilización es el mecanismo de fuga más importante para aplicaciones en V6 (Prystupa, 2007).

Se llama rendimiento objetivo a la técnica de fertilización en función de un rendimiento esperado y determinado fundamentalmente en base a la oferta de nitratos a la siembra y demanda de nitrógeno por cada tonelada de cultivo esperado a cosecha. Las aplicaciones en V6 presentan mayores rendimientos y nitrógeno acumulado en el vegetal debido a menores pérdidas por lixiviación (Prystupa, 2007).

En cuanto a la demanda de nutrientes, la gran cantidad se da a partir de V5 (20 a 30 días posteriores a la emergencia) y hasta 10 días previos a la floración, período en el cual el cultivo toma aproximadamente el $70 \%$ de los nutrientes requeridos. Por lo tanto una buena nutrición desde los primeros estadios de desarrollo producirá una cantidad de área foliar suficiente para interceptar la mayor parte de la radiación incidente y asegurar así una alta eficiencia para transformarla en biomasa (Bernardis et al 2002).

El principal componente del rendimiento es el número de panojas por ha, es decir mayor número de plantas por ha, en siembra directa a $52 \mathrm{~cm}$. entre líneas un stand de $140.000 \mathrm{pl} \mathrm{x} \mathrm{ha}^{-1}$ es adecuado, dependiendo de las condiciones ambientales y fecha de siembra (Chessa, 2008).

Considerando el uso del agua, es posible la producción de granos con $250 \mathrm{~mm}$ de agua en su ciclo, pero con $450 \mathrm{~mm}$ se obtendrán los mejores rendimientos según el híbrido y fecha de siembra (Chessa, 2008).

En este trabajo, se plantea estudiar el efecto de la fertilización con urea en estadio de V6, seis hojas verdaderas desplegadas en sorgo granífero para un rendimiento objetivo previsto en un sistema de siembra directa del Suroeste de la provincia de Chaco.

En la región se carece de información de las relaciones entre la oferta de nitratos a la siembra, agua en el perfil y el efecto de la fertilización sobre un rendimiento esperado en cereales de verano.

\section{MATERIALES Y MÉTODOS}

El ensayo se realizó en la Estación Experimental Agropecuaria INTA Las Breñas, ubicada a los $27^{\circ} 04^{\prime} 70^{\prime \prime}$ Sur y $61^{\circ} 03^{\prime} 30^{\prime \prime}$ Oeste y a los 101,6 metros sobre el nivel del mar.

El suelo correspondió a la Serie Tizón, Haplustol óxico, familia limosa fina, mixta hipertérmica. Todo 
el perfil de textura pesada. Moderadamente pobre en materia orgánica, medianamente alta capacidad de retención de agua hasta los $160 \mathrm{~cm}$. de profundidad, alto contenido de fósforo, rico en calcio y magnesio y muy rico en potasio, con moderadamente alta capacidad de intercambio catiónico y alto porcentaje de saturación de bases. El Horizonte A varía de 18 a 27 $\mathrm{cm}$. de espesor, textura media; el B entre 45 a $55 \mathrm{~cm}$., textura pesada; el $\mathrm{C}$ se presenta a partir de los 44 a 80 $\mathrm{cm}$ de profundidad (Ledesma y Zurita, 2004).

El lote destinado al ensayo se encontraba bajo sistema de siembra directa teniendo como cultivo antecesor maíz. La siembra fue realizada el día 22 de enero utilizando el cultivar Morgan 109 de ciclo intermedio, con una sembradora PLA de siembra directa de 8 surcos. El diseño experimental utilizado fue en Bloques Completamente Aleatorizado con 4 repeticiones.

Las unidades experimentales fueron de $18,72 \mathrm{~m}^{2}$ (6 $\mathrm{m}$ de largo por 3,12 $\mathrm{m}$ de ancho). La distancia entre surcos fue de $52 \mathrm{~cm}$ y se logró una densidad de 100.000 plantas $\mathrm{ha}^{-1}$, Se realizaron las aplicaciones de agroquímicos recomendadas por INTA para la protección del cultivo.

La humedad edáfica fue determinada a la siembra, utilizando el método gravimétrico $(\% \mathrm{P} / \mathrm{P})$ tomando muestras cada $20 \mathrm{~cm}$ hasta la profundidad de $1 \mathrm{~m}$. Así también, se determinó la densidad aparente, por el método del cilindro de Blake y Hartage (1986), con posterior determinación de lámina de agua disponible.

Para la fertilización, se utilizó como fuente nitrogenada urea a razón de $30 \mathrm{~kg} \mathrm{x} \mathrm{ha}^{-1}$ para el tratamiento T1 y $90 \mathrm{~kg}$ x ha. ${ }^{-1}$ para T2 para un Rendimiento Objetivo esperado de $8.000 \mathrm{~kg}$ de grano $\mathrm{x} \mathrm{ha}^{-1}$ y $10.000 \mathrm{~kg}$ de grano $\mathrm{xha}^{-1}$, respectivamente. La fertilización se efectuó en el estado V6 (6 hojas verdaderas desplegadas) en la línea de siembra, en forma manual, con incorporación del fertilizante. Se determinó el contenido de nitratos de 0 a $20 \mathrm{~cm}$ a la siembra y al momento de la fertilización, para mejor ajuste de la dosis y de los requerimientos para alcanzar rendimientos objetivos de 8.000 $\mathrm{kg}$ y $10.000 \mathrm{~kg} \mathrm{x} \mathrm{ha}^{-1}$. El rendimiento objetivo se calcula en función de una demanda a razón de $30 \mathrm{~kg}$ de urea por cada $1000 \mathrm{~kg}$ de rendimiento de grano con $0 \%$ de humedad de grano (IPNI, 2007). Con la información determinada de densidad aparente, nitratos a la siembra y una profundidad dada se pasó nitratos a $\mathrm{kg}$. de urea por ha de oferta a la siembra. Como determinaciones complementarias se midió también el contenido de fósforo y potasio.
Las muestras para la cosecha fueron recolectadas manualmente en $1 \mathrm{~m}$ lineal y desgranadas en una trilladora estática. La humedad del grano fue obtenida por secado en estufa hasta peso constante a $80^{\circ} \mathrm{C}$, estimándose el rendimiento en grano con $0 \%$ de humedad. Se determinó biomasa aérea (rastrojo y grano), peso de rastrojo, índice de cosecha, uso consuntivo, eficiencia de uso de agua y eficiencia de uso de fertilizante y relación ingreso bruto costo fertilizante.

Se realizó un Análisis de Varianza de los parámetros evaluados, con el Test de Tukey se identificaron las diferencias significativas entre los tratamientos. Se realizó análisis de regresión con los rendimientos obtenidos. Se utilizó el software estadístico INFOSTAT ver. P. 2007.

\section{RESULTADOS Y DISCUSIÓN}

El análisis a la siembra de la disponibilidad de nitratos del suelos (Tabla $\mathrm{N}^{\circ} 1$ ), indicó que el mismo estuvo bien provisto, $38 \mathrm{ppm}$, a pesar de estar dedicado a la agricultura extensiva en sistema de Labranza Cero, desde hace 5 años. Para la fertilización nitrogenada en cereales de verano, no se recomienda hacerla a la siembra por estar fuera del umbral de respuesta 18 a $20 \mathrm{ppm}$, ofreciendo bajas posibilidades de respuesta económica a la fertilización (Melgar y Torres Duggan, 2007). Por este motivo, la concentración de nitratos a la siembra fue un indicador de la no conveniencia de fertilizar a la siembra.

Los valores de nitratos, indican un suelo bien provisto $38,03 \mathrm{ppm}$ y $65,88 \mathrm{ppm}$ en fósforo (Tabla 1 ). Para los suelos de la región son valores normales y no manifiestan signos evidentes de deficiencia en los cultivos.

El haber realizado un barbecho largo, en siembra directa permitió acumular 70,2 $\mathrm{mm}$ hasta $1 \mathrm{~m}$ de profundidad y llegar a la fecha límite de siembra por ocurrencia de heladas tempranas, el 15 de abril para los últimos cuatro años, de acuerdo a los datos provistos por la Estación Agrometeorológica INTA Las

Tabla $\mathbf{N}^{0}$ 1: Fertilidad química del suelo a la siembra.

\begin{tabular}{l|ccc}
\hline & $\begin{array}{c}\text { Nitratos (NO3) } \\
\mathbf{p p m}\end{array}$ & $\begin{array}{c}\text { Fósforo (P) } \\
\mathbf{p p m}\end{array}$ & $\begin{array}{c}\text { Potasio (K) } \\
\text { meq/100 g suelo }\end{array}$ \\
\hline Media & 38,03 & 65,88 & 0,53 \\
Desv. Estandar & 2,77 & 8,10 & 0,02 \\
C.V. & 7,27 & 12,30 & 3,44 \\
\hline
\end{tabular}

Tabla $N^{0}$ 2: Eficiencia del uso del agua por grano producido.

\begin{tabular}{cc}
\hline Tratamientos & $\begin{array}{c}\text { Eficiencia de Uso de Agua (EUA) } \\
\text { kg grano. } \text { mm agua }^{-1}\end{array}$ \\
\hline T0 & 16,4 \\
T1 & 24,4 \\
T2 & 28,3 \\
\hline
\end{tabular}


Tabla No 3: Análisis de Varianza, test de Tukey y parámetros para índice de cosecha, rendimiento en grano seco, biomasa, peso de rastrojo.

\begin{tabular}{|c|c|c|c|c|c|}
\hline Tratamiento & Parámetros & $\begin{array}{l}\text { Índice de } \\
\text { cosecha }\end{array}$ & $\begin{array}{l}\text { Rendimiento en grano } \\
\text { seco }\left(\mathrm{kg} \times \mathrm{ha}^{-1}\right)\end{array}$ & $\begin{array}{l}\text { Biomasa aerea } \\
\left(\mathrm{kg} \mathrm{x} \mathrm{ha}^{-1}\right)\end{array}$ & $\begin{array}{l}\text { Peso de Rastrojo } \\
\left(\mathrm{kg} \mathrm{x} \mathrm{ha}^{-1}\right)\end{array}$ \\
\hline \multirow{2}{*}{ T 0} & Media & $0,29(a)$ & $4.942,11(\mathrm{a})$ & $17.667,56(a)$ & $12.725,45$ (a) \\
\hline & Desv. Estandar & & 498,50 & $2.468,11$ & $2.775,05$ \\
\hline \multirow{2}{*}{ T 1} & Media & $0,34(a)$ & $7.345,86(b)$ & $21.393,38(a b)$ & $14.047,52(a b)$ \\
\hline & Desv. Estandar & & $1.569,18$ & $2.820,22$ & $1.512,58$ \\
\hline \multirow{2}{*}{ T 2} & Media & $0,34(a)$ & $8.518,89(b)$ & $25.287,45$ (b) & $16.768,56(b)$ \\
\hline & Desv. Estandar & & 485,34 & 886,46 & 730,23 \\
\hline
\end{tabular}

Letras distintas indican diferencias significativas $(p \leq 0.05)$

Breñas, con una humedad adecuada, medida el 12 de enero de 2008 , con un porcentaje de humedad a saturación de $27 \%$ y una densidad aparente promedio de 0 a $40 \mathrm{~cm}$. de $1,49 \mathrm{gr} . / \mathrm{cm} 3$.

La precipitación en el ciclo del cultivo fue de $231,5 \mathrm{~mm}$., con lo que el agua usada por el cultivo en el ciclo fue de $301,5 \mathrm{~mm}$ existiendo a $1 \mathrm{~m}$. de profundidad $0 \%$ de humedad.

Para el Ciclo 2007-2008, año de sequía, se registraron valores de défict hídrico en 11 meses, solo se registró exceso en el mes de diciembre. La Evapotranspiración Potencial Anual fue de $1418 \mathrm{~mm}$ y las precipitación de julio 2007 a junio 2008 de $644 \mathrm{~mm}$ (Figura $\mathrm{N}^{\mathrm{o}}$ 1.).

La eficiencia en el uso de un recurso (radiación, nitrógeno) representa la cantidad de producto logrado (materia seca o granos) por unidad de recurso capturado por el cultivo (Hunt, 1990, citado por Caviglia et al. 2007). En consecuencia, la mayor productividad de un cultivo puede lograrse por una mayor captura de recursos, por una mayor eficiencia en su uso o por ambas a la vez.

La fertilización permitió hacer más eficiente el uso del agua, lográndose $28,3 \mathrm{~kg}$ de grano por $\mathrm{mm}$ de agua utilizada en el tratamiento $\mathrm{T} 2$, contra $16,4 \mathrm{~kg}$ de grano por mm de agua, en T0 (Tabla $\mathrm{N}^{\circ}$ 2).

En la Tabla $\mathrm{N}^{\circ} 3$ es posible apreciar los parámetros estadísticos del índice de cosecha, rendimiento en grano, biomasa y peso de rastrojo, todos referidos expresados en peso seco. García et al (2001) al fertilizar a la siembra sorgo granífero de ciclo corto con iguales dosis de nitrógeno que en el presente trabajo y sembrado en setiembre,

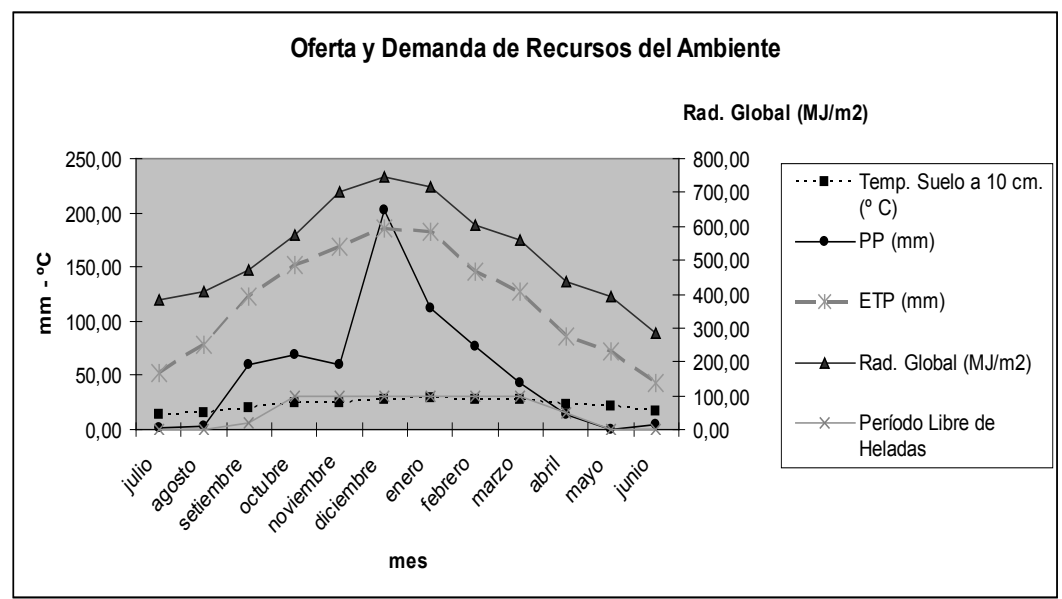

Figura $\mathbf{N}^{0}$ 1: Distribución mensual de la oferta y demanda del ambiente. Elaboración propia en base a datos de la Estación Meteorológica EEA INTA Las Breñas, 2008.

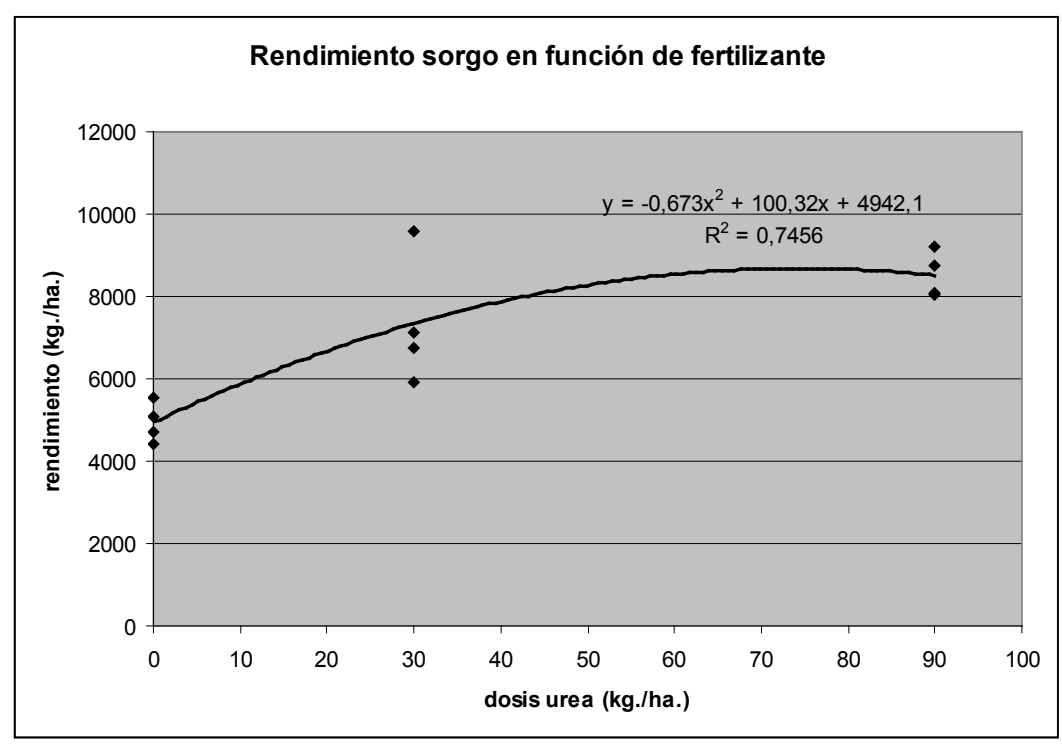

Figura $\mathbf{N}^{\circ}$ 2: Ecuación de regresión: rendimiento del sorgo granífero logrado en función de la dosis de urea aplicada. 
Tabla $\mathbf{N}^{0}$ 4: Eficiencia del uso de Urea en función de la dosis aplicada.

\begin{tabular}{|c|c|c|c|c|}
\hline Tratamiento & $\begin{array}{l}\text { Dosis fert. Urea } \\
\quad\left(\mathrm{kg} \mathrm{.ha}^{-1}\right)\end{array}$ & $\begin{array}{l}\text { Rendimiento promedio } \\
\left(\mathbf{k g} \cdot \mathrm{ha}^{-1}\right)\end{array}$ & 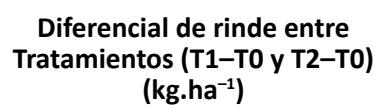 & $\begin{array}{l}\text { Eficiencia de uso } \\
\left(\mathrm{kg}^{2} \text { grano. } \mathrm{kg} \mathrm{urea}^{-1}\right)\end{array}$ \\
\hline TO & 0 & 4942,11 & & \\
\hline $\mathrm{T} 1$ & 30 & 7345,86 & 2403,75 & 80,1 \\
\hline $\mathrm{T} 2$ & 90 & 8518,89 & 3576,78 & 39,7 \\
\hline
\end{tabular}

Tabla No 5: Relación Ingreso-Costo de uso de fertilizante nitrogenado.

\begin{tabular}{ccccc}
\hline Tratamientos & $\begin{array}{c}\text { Dosis de fertilizante } \\
\text { urea }\end{array}$ & $\begin{array}{c}\text { Rendimiento } \\
\text { promedio }\end{array}$ & $\begin{array}{c}\text { Costo urea por dosis/ha } \\
\text { (\$) }\end{array}$ & $\begin{array}{c}\text { Ingreso bruto-costo urea } \\
\text { (\$) }\end{array}$ \\
\hline T0 & 0 & $4.942,11$ & 0 & 2.223 \\
T1 & 30 & $7.345,86$ & 48 & 3.255 \\
T2 & 90 & $8.518,89$ & 144 & 3.681 \\
\hline
\end{tabular}

para el Noreste de provincia de Chaco, obtuvieron rendimientos inferiores para ambas dosis utilizadas. Esto estaría indicando la mayor eficiencia en el uso del fertilizante en V6, con hojas totalmente desplegadas, aunque el cultivar de ciclo intermedio explicaría un rendimiento menor al potencial para el experimento, sumado a escasas precipitaciones en el ciclo del cultivo.

Existe una disminución de la eficiencia agronómica del uso de urea en la medida que aumenta la dosis de fertilizante, pasando de $80,1 \mathrm{~kg}$ de grano a $39,7 \mathrm{~kg}$ de grano logrado por $\mathrm{kg}$ de urea aplicada (Tabla 4).

Bernardis, et al (2002) para un ensayo de fertilización nitrogenada realizado en la provincia de Corrientes en un sorgo granífero en siembra directa, mencionan que la mayor producción de grano y materia seca se produjo con las dosis de fertilizante de 60 y $90 \mathrm{~kg}$. ha. ${ }^{-1}$ en forma de urea.

En la Ecuación de Regresión de la Figura $\mathrm{N}^{0} 2$, se observa la respuesta del rendimiento a la dosis de fertilizante, la misma, responde a una curva de tipo polinómica de segundo grado con un $\mathrm{R}^{2}$ de 0,74 que indica un buen ajuste.

Por su parte, Castaneda et al (2006) informan una ecuación cuadrática para la fertilización nitrogenada de arranque y a los 30 días de emergencia y similar a la informada por García et al (2001) con fertilización a la siembra.

Caviglia, et al (2007) menciona la misma tendencia observada en la Figura $\mathrm{N}^{\mathrm{o}} 2$. Ante un incremento de la disponibilidad de nitrógeno, la eficiencia del uso del nitrógeno disminuye.

En la Tabla $N^{\circ} 5$, se refleja que para el mes de mayo de 2008 con un precio del sorgo granífero de U\$S144/ tn, precio pizarra de Rosario y el precio de urea de USS 514/tn., para el mismo mes, la relación ingresocosto resultó positiva, recomendando la práctica para lograr un mejor aprovechamiento de los recursos que brinda el ambiente: agua, radiación, suelo, días que dura el ciclo del cultivo y beneficio económico.

\section{CONCLUSIONES}

La concentración de nitratos a la siembra permitió diferir la aplicación del $100 \%$ de la dosis de fertilizante (urea) en V6, obteniéndose una eficiente respuesta de la planta a la fertilización nitrogenada.

La metodología de Rendimiento Objetivo resultó adecuada para estimar la dosis de fertilizante a aplicar y los rendimientos esperados.

La relación ingreso-costo resultó positiva para recomendar la práctica al momento de su aplicación.

La fertilización nitrogenada permitió mejorar la eficiencia del uso del agua a partir de los recursos que brindó el ambiente.

En base a los alentadores resultados obtenidos y teniendo presente las condiciones ambientales extremas en las que se realizó el ensayo, es necesario continuar con este tipo de experimentos para lograr a generalizaciones regionales.

\section{AGRADECIMIENTOS}

Este trabajo pudo realizarse gracias al financiamiento otorgado por el Proyecto Integrado de INTA PNCER 2. Tecnologías para la producción sustentable de cereales y oleaginosas en sistemas agropecuarios de la región pampeana y extra pampeana. Proyecto Específico: Rotaciones y labranzas: Sistemas agrícolas de alta productividad (PNCER 2341) y los aportes del Ing. Marcelo Bodrero.

\section{BIBLIOGRAFÍA}

Bernardis, H.O.; García P.A. y Ferrero, A.R. 2002. Estructura del cultivo, fertilización nitrogenada, radiación interceptada y producción de materia seca en sorgo (Sorghum bicolor L. moench) en siembra directa. Jornadas de Comunicación Científicas y Tecno- 
lógicas. Universidad Nacional del Nordeste. Corrientes. A-079.

Blake, G. and Hartge, K. 1986. Bulk density. In: Methods of soil analysis. Part 1. SSA Books Series. 59. Editor Klute. Am. Society of Agronomy, Inc. Soil Sci. Soc. Am., Inc. Madison, Wisconsin, USA. pp. 630.

Boletín Agrometeorológico annual. 2008. EEA INTA Las Breñas, Chaco.

Castaneda, J.W.; Mason, S.; Argueta Portillo, Q.; Elías, R. .; Hernández, M. y Valencia, R.C. 2006. Eficiencia del uso de nitrógeno en sorgo sensible al fotoperíodo en asocio con maíz en el Salvador. Agronomía Mesoamericana 17 (2): 11-17.

Caviglia, O.P.; Melchiori R.J. M.; Kemerer A.; Van Opstal N.V. y Gregorutti, V.C. 2007. Relaciones entre la eficiencia en el uso del nitrógeno y de la radiación en maíz. Actualización Técnica en Maíz, girasol y Sorgo. EEA Paraná. Serie Extensión No 44. Agosto de 2007.

Chessa, A. 2008. Pensando la siembra de sorgo granífero. Información técnica cultivos de verano. Campaña 2007. INTA EEA Rafaela. Publicación Miscelanea $\mathrm{N}^{\mathrm{o}}$ 108.

Dirección de Estadística de la provincia de Chaco. 2006. Resistencia. Chaco. ISSN 0577-4861

EEA INTA Las Breñas. 2008. Boletín Agrometeorológico anual 2008.

García, Pedro A.; Ferrero, Abel R. y M. Goujon. 2001. Fertilización nitrogenada en siembra directa de sorgo. Jornadas de Comunicación Científicas y Tecnológicas. Universidad Nacional del Nordeste. Corrientes. A-066.

Hunt. 1990. Citado por Caviglia, O. P.; Melchiori R.J.M.; Kemerer A.; Van Opstal N.V. y Gregorutti, V.C. 2007. Relaciones entre la eficiencia en el uso del nitrógeno y de la radiación en maíz. Actualización Técnica en Maíz, girasol y Sorgo. EEA Paraná. Serie Extensión No 44. Agosto de 2007.
INFOSTAT. 2007. Manual del Usuario. Universidad Nacional de Córdoba. Córdoba, Argentina.

IPNI. 2007. Planilla de cálculo para estimar requerimientos nutricionales de cultivos de cereales, oleaginosos, forrajeras e industriales. CalReq2007. IPNI Cono Sur. International Plant Nutrition Institute. Ultima revisión 12 de Julio de 2007.

Ledesma, L.L. y Zurita, J.J. 2004. Carta de suelos de la República Argentina. Provincia del Chaco. Los suelos del departamento 9 de Julio. Convenio INTA; Gobierno de la provincia del Chaco, Ministerio de la Producción.

Melgar, R. y Torres Duggan, M. 2007. Manejo de la fertilización en maíz. IDIA 6. Bs. As. Ediciones INTA. IDIA XXI.

Melín, A. A. y Zamora, M. 2007. Tecnología del Cultivo. Cap. 4. Sorgo en el Sur. Editores Martín s. Zamora y Ariel A. Melín. Ediciones INTA, Ministerio de Asuntos Agrarios, gobierno de la provincia de Buenos Aires. $1^{\circ} \mathrm{Ed}$. Bs. As.

Pagani, A.; Echeverría, H.E.; Sainz Rozas, E.R. y Barberi, P.A.. 2008. Dosis óptima económica de nitrógeno en maíz bajo siembra directa en el Sudeste bonaerense. XXI Congreso Argentino de la Ciencia del Suelo. Potrero de Los Funes, San Luis.

Prystupa, P. 2007. Tecnología de la fertilización de cultivos extensivos en la región pampeana. EUDEBA. Bs. As.

Secretaría de Agricultura, Ganadería, Pesca y Alimentos. 2009. Estimaciones agrícolas mensuales. Cifras Oficiales al 21/01/09.

http://www.sagpya.gov.ar/new/0-0/agricultura/otros/estimaciones/pdfmensual/enero_2009.pdf

Vanderlip R.L. 1993. How a sorghum plant develops. Cooperative extension service. Contribution No 1203. Kansas Agricultural Experiment Station, Manhattan, Kansas. USA. 